\title{
DIFFERENTIAL INEQUALITIES FOR HYSTERESIS SYSTEMS ${ }^{1}$
}

\author{
VLADIMIR V. CHERNORUTSKII and \\ MARK A. KRASNOSEL'SKII \\ Institute for Information Transmission Problems of RAS \\ 19, Bol'shoi Karetnyi pereulok, 101447, Moscow Russia \\ chernoru@nov.ippi.ras.ru and amk@ippi.ac.msk.su
}

(Received April, 1996; Revised August, 1996)

\begin{abstract}
The theory of differential inequalities is extended to functional-differential equations with hysteresis nonlinearities. A key feature is the existence of a semiorder of the state space of nonlinearity and a special monotonicity of the righthand side of differential inequality.

This article is dedicated to the memory of Roland L. Dobrushin.

Key words: Differential Inequality, Hysteresis Nonlinearity, State Space, Monotonicity, Initial Value Problem.
\end{abstract}

AMS (MOS) subject classifications: $34 \mathrm{~A} 40,47 \mathrm{H} 30$.

\section{Introduction}

In this paper, functional-differential inequalities of the kind

$$
x^{\prime}(t)<f(t, x(t), \xi(t))
$$

are considered, where $\xi(t)$ stands for the output of a hysteresis transducer (or in other words, that of a hysteresis nonlinearity) corresponding to the input $x(t)$. The transducer is regarded as a dynamical system which may have different states and whose evolution in time is determined by input-state and state-output operators.

The study of differential inequalities dates back to Tchaplygin. Over the years an abundance of work was contributed to this topic. Among most notable are fundamental monographs by Szarski [7] and Lakshmikantham and Leela [5]. The latter monograph is particularly concerned with functional-differential inequalities.

In the present paper, a new type of inequalities is studied. This is characterized by the dependence of the right-hand side upon the output of the hysteresis nonlinearity. The results obtained are applied to specified classes of nonlinearities.

\subsection{Hysteresis transducers}

In what follows, $W$ will be a deterministic transducer (or, in other words, a dynamical sys-

\footnotetext{
${ }^{1}$ The work was reported on Dobrushin's Laboratory Seminar at IITP of RAS.
} 
tem) having continuous input $x(t)$ and output $\xi(t)$. We will assume that the state space $\Omega(\mathcal{W})$ of the transducer $\mathcal{W}$ consists of pairs $\omega=(x, q)$ whose first components are scalar-valued and the second ones belong to a set $Q$ which may depend on $x$. The property of the transducer to be deterministic means that for any instant of time $t_{0}$, any initial state $\omega_{0}=\left(x_{0}, q_{0}\right) \in \Omega(\mathcal{W})$, and any admissible input $x(t)$, there exists the evolution law

$$
q(t)=Z\left[t_{0}, \omega_{0}\right] x(t) \quad\left(t \geq t_{0}\right)
$$

of the second component of the state $\omega(t)=(x(t), q(t))$ for the transducer $\mathcal{W}$. The admissibility of the input $x(t)$ means that the function $x(t)$ is continuous for $t \geq t_{0}$ and obeys the condition $x\left(t_{0}\right)=x_{0}$.

As usual, in systems theory $[2,8]$, the family of the operators $Z\left[t_{0}, \omega_{0}\right]$ describing the inputoutput mapping for the transducer $W$ should satisfy the semigroup identity. Specifically, if an input $x(t)$ is admissible, i.e., function (1) is well-defined, then the operator $Z\left[t_{1}, \omega_{1}\right]$ is also well-defined for the input with $t_{1} \geq t_{0}, \omega_{1}=\left\{x\left(t_{1}\right), q\left(t_{1}\right)\right\}$ and

$$
Z\left[t_{1}, \omega_{1}\right] x(t) \equiv Z\left[t_{0}, \omega_{0}\right] x(t) \text { for } t \geq t_{1} .
$$

The operator $Z$ is also called the transition operator.

The value of the output signal at any instant is completely determined by the state of the transducer at the same instant, i.e.,

$$
\xi(t)=F[\omega(t)]=F\left[x(t), Z\left[t_{0}, \omega_{0}\right] x(t)\right] .
$$

Instead of the latter equality, we will also write

$$
\xi(t)=W\left[t_{0}, \omega_{0}\right] x(t) .
$$

The image of the set $\Omega(\mathcal{W})$ with respect to the mapping $F$ will be denoted by $\mathscr{F}(F)$.

We assume that the operators $W\left[t_{0}, \omega_{0}\right]$ of the input-output correspondence defined and continuous on the spaces $C\left(t_{0}, t_{1}\right)$ of continuous functions on a segment $\left[t_{0}, t_{1}\right]$ where $t_{0}$ and $t_{1}$ are arbitrary numbers such that $t_{0}<t_{1}$. For the sake of brevity, these spaces will be denoted by $C$.

\subsection{Examples}

1. Generalized play and hysteron. Let two curves be given which are the graphs of monotonically nondecreasing functions $q=\Gamma_{l}(x)$ and $q=\Gamma_{r}(x)$ such that $\Gamma_{r}(x) \leq \Gamma_{l}(x)$. The state space of the generalized play $\mathcal{L}$ is the strip

$$
\Omega(\mathcal{L})=\left\{(x, q) \mid \Gamma_{r}(x) \leq q \leq \Gamma_{l}(x)\right\} .
$$

First define operator (1) for monotone continuous inputs $x(t)$ as follows:

$$
Z\left[t_{0}, \omega_{0}\right] x(t)= \begin{cases}\max \left\{q_{0}, \Gamma_{r}(x(t))\right\}, & \text { for } x(\cdot) \text { not decreasing } \\ \min \left\{q_{0}, \Gamma_{l}(x(t))\right\}, & \text { for } x(\cdot) \text { not increasing. }\end{cases}
$$

For piecewise monotone inputs $x(t)$, define the operator of the generalized play by sequential application of equation (5) and using semigroup identity (2). The set of piecewise monotone functions is dense everywhere in the space $C$. It turns out (see [4]) that the operator $Z$ can be extended by continuity in $C$ onto the set of all continuous inputs. As it was shown in [4], the operators $Z\left[t_{0}, \omega_{0}\right]$ for the generalized play are well-defined and continuous on the space $C$.

The output of the generalized play coincides with the second component of the state, i.e., operators (1) and (4) coincide.

To define a hysteron, introduce along with the functions $\Gamma_{r}$ and $\Gamma_{l}$ a continuous function $\alpha(x, z)$ which is continuous in its variables and strictly monotone in $z$ for any fixed $x$. The state 
space of the hysteron $\mathscr{W}$ is the set

$$
\Omega(\mathscr{W})=\left\{(x, q) \mid q \in\left[\alpha\left(x, \Gamma_{r}(x)\right), \alpha\left(x, \Gamma_{l}(x)\right)\right]\right\}
$$

(here, $\left[\beta_{1}, \beta_{2}\right]$ denotes either the closed segment $\left[\beta_{1}, \beta_{2}\right]$ of the real axis if $\beta_{1} \leq \beta_{2}$ or segment $\left[\beta_{2}, \beta_{1}\right]$ if $\left.\beta_{1}>\beta_{2}\right)$. Put

$$
Z\left[t_{0}, \omega_{0}\right] x(t)=\alpha\left(x(t), W_{\mathcal{L}}\left[t_{0}, \omega_{0}\right] x(t)\right),
$$

where $W_{\mathcal{L}}$ is the input-output operator of the generalized play. Therefore, the functions $\Gamma_{r}, \Gamma_{l}$, and $\alpha$ completely determine some hysteron.

The generalized play is a special case of the hysteron. If $\Gamma_{r}(x)=x-h, \Gamma_{l}(x)=x+h$, where $h$ is a positive constant, and $\alpha(x, z)=z$, then the hysteron is called the ordinary play (with the gap 2h). If $\alpha(x, z)=x-z$ then such a hysteron is called the stop (with the threshold values $\pm h$ ) and is used as a model for the ideal elasto-plastic fibre. (It is nothing but the Prandtl model.)

The input-output operators of the hysteron are continuous on the space $C$. Under quite general assumptions ([4], p. 40), these operators satisfy the Lipschitz condition.

2. Ishlinskii model. The model is used in elasto-plasticity theory. Let a bounded-variation function $\mu(\cdot)$ be given on the segment $\Pi=[0, H]$. The state space of the Ishlinskii transducer is the set

$$
\Omega=\{(x, q(\cdot)) \mid \text { function } q(\cdot) \text { is } \mu \text {-measurable and } \forall h \in \Pi \quad|q(h)| \leq h\} .
$$

Let $\omega_{0}=\left(x_{0}, q_{0}(\cdot)\right) \in \Omega$. The transition operator $Z$ of the Ishlinskii transducer assigns a function $q(\cdot)(t)$ to a pair $\left(\omega_{0}, x(t)\right)$ in such a way that for any $h \in \Pi$,

$$
q(h)(t)=Z_{h}\left[t_{0},\left(x_{0}, q_{0}(h)\right)\right] x(t),
$$

where $Z_{h}$ is the input-output operator of the stop with the threshold values $\pm h$. Therefore, the state of the Ishlinskii transducer is a one-parametric family of states of stops with different threshold values.

The output of the Ishlinskii transducer is defined as a Lebesgue-Stieltjes integral of the states of the stops

$$
F[x(t), q(\cdot)(t)]=\int_{\Pi} q(h)(t) d \mu(h) .
$$

3. Relay and Preisach model. Let $\alpha$ and $\beta$ be real numbers such that $\alpha<\beta$. The state space of the nonideal relay $\mathscr{R}_{\alpha, b}$ is given by the set

$$
\left.\Omega\left(\Re_{\alpha, \beta}\right)=\{(x, 0)\} x<\beta\right\} \cup\{(x, 1) \mid x>\alpha\} .
$$

Denote by $T_{\gamma}\left(t_{1}\right)$ the set of instants $t \in\left[t_{0}, t_{1}\right]$ such that $x(t)=\gamma$ and $x(s) \in(\alpha, \beta)$ for any $s \in\left[t, t_{1}\right]$. The transition operator $Z_{(\alpha, \beta)}$ is given by

$$
Z_{(\alpha, \beta)}\left[t_{0}, \omega_{0}\right] x(t)=\left\{\begin{aligned}
q_{0}, & \text { if } T_{\gamma}(t)=\emptyset \text { for all } \gamma \notin(\alpha, \beta) ; \\
1, & \text { if } x(t) \geq \beta \text { or } T_{\beta}(t) \neq \emptyset \\
0, & \text { if } x(t) \leq \alpha \text { or } T_{\alpha}(t) \neq \emptyset .
\end{aligned}\right.
$$

One can also say that the second component of the state of the nonideal relay corresponding to the input $x(t)$ is a piecewise constant function $q(t)$ taking on either 0 or 1 and having the minimal variation among all the functions satisfying the condition

$$
\omega(t)=(x(t), q(t)) \in \Omega\left(\mathscr{R}_{\alpha, \beta}\right) \text { for all } t \in\left[t_{0}, t_{1}\right] .
$$

The zero and unit values of the state can be interpreted as "switched-off" and "switched-on" positions of the relay. 
Now, let us turn to the Preisach model which is often used in magnetics theory [6]. Let a measure $\mu$ be given on the half-plane $\Pi=\{(\alpha, \beta) \mid \alpha<\beta\}$ with a continuous density. The state space of the Preisach transducer 90 is the set

$$
\Omega(\mathscr{P})=\left\{(x, q(\cdot, \cdot)) \mid \forall(\alpha, \beta) \in \Pi \quad(x, q(\alpha, \beta)) \in \Omega\left(\mathscr{R}_{\alpha, \beta}\right)\right\},
$$

where $q(\cdot, \cdot)$ is a $\mu$-measurable function. The transition operator $Z$ of the Preisach transducer maps a pair $\left(\omega_{0}, x(t)\right)$ to the function $q(\cdot, \cdot)(t)$ such that for any $(\alpha, \beta) \in \Pi$,

$$
q(\alpha, \beta)(t)=Z_{(\alpha, \beta)}\left[t_{0}, \omega_{0}\right](t)
$$

where $Z_{(\alpha, \beta)}$ is given by equation (6). Therefore, the Preisach transducer can be regarded as a two-parametric family of nonideal relays. Similarly to what was done when describing the Ishlinskii model, the output of the Preisach transducer is defined as the integral of the states of non ideal relays with respect to the measure $\mu$

$$
F[x(t), q(\cdot, \cdot)(t)]=\int_{\Pi} q(\alpha, \beta)(t) d \mu(\alpha, \beta) .
$$

Note that for all $\alpha, \beta$, the function $q(\alpha, \beta)(t)$, which is the output of the corresponding relay, is, in general, discontinuous in $t$. Nevertheless (see [4]), the output of the Preisach transducer is a continuous function and moreover the corresponding hysteresis operator is well-defined on space $C$.

\section{Initial Value Problem}

In what follows, we will consider the functional-differential equation

$$
\begin{aligned}
& x^{\prime}(t)=f(t, x(t), \xi(t)) \\
& \xi(t)=W\left[t_{0}, \omega_{0}\right] x_{1}(t),
\end{aligned}
$$

where $x(t) \in \mathbb{R}^{n}$, the function $f: \mathbb{R} \times \mathbb{R}^{n} \times \mathscr{F}(F) \rightarrow \mathbb{R}^{n}$ is continuous in its variables, $\xi(t)$ is the output of the hysteresis nonlinearity $W$ corresponding to the input $x_{1}(t)$ which is the first component $x_{1}(t)$ of the function $x(t)$.

To specify individual solutions of equation (7) we give the initial values

$$
x\left(t_{0}\right)=x_{0}, \quad \omega\left(t_{0}\right)=\omega_{0} \in \Omega(W)
$$

with $\omega_{0}=\left(x_{1}\left(t_{0}, q\left(t_{0}\right)\right)\right.$.

A continuous differentiable function $x(t)$ satisfying relations (7)-(9) is called the solution of the initial value problem for the differential equation with hysteresis nonlinearity.

With the aid of standard tools (for example, by using the Schauder principle for contraction mappings), one can easily prove the validity of the following statements:

- The initial value problem (7)-(9) has at least one solution defined on a segment $\left[t_{0}, t_{0}+\epsilon\right]$.

- If operator (4) satisfies on $C\left(t_{0}, t_{1}\right)$ the local Lipschitz condition (as in the case of wide classes of hysterons, Ishlinskii and Preisach transducers, etc.) and the function $f(t, x, \xi)$ obeys the local Lipschitz condition in its variables $x$ and $\xi$, then initial value problem (7)(9) has the unique solution.

More general results on solvability and uniqueness of solution of initial value problems are given in [1]. 


\section{Directed Nonlinearities and Special Monotonicity}

\subsection{Directed nonlinearities}

The state space $\Omega(\mathcal{W})$ of a transducer $\mathcal{W}$ will be endowed with semiorder denoted by the symbol $\prec$.

Definition 1: A transducer $\mathscr{W}$ is called directed if for any admissible inputs $u(t)$ and $v(t)$ and for any initial states $\omega_{1}=\left(u\left(t_{0}\right), q_{1}\right)$ and $\omega_{2}=\left(u\left(t_{0}\right), q_{2}\right) \in \Omega(\mathcal{W})$, the relations

$$
\omega_{1} \prec \omega_{2}, \quad u(t) \leq v(t) \quad\left(t \in\left[t_{0}, t_{1}\right]\right)
$$

imply the validity of the relations

$$
\left(u(t), Z\left[t_{0}, \omega_{1}\right] u(t)\right) \prec\left(v(t), Z\left[t_{0}, \omega_{2}\right] v(t)\right)
$$

for all $t \in\left[t_{0}, t_{1}\right]$.

The latter means that $\omega_{1}(t) \prec \omega_{2}(t)$ remains valid for all $t$ under consideration.

Examples: To make sure that a hysteresis transducer possesses directness one should first introduce an appropriate semiorder on the state space of the transducer.

1. If $\mathcal{L}$ is the generalized play then the following semiorder on the set $\Omega(\mathcal{L})$ can be employed:

if $q_{0} \leq q_{1}$.

$$
\omega_{0}=\left(x_{0}, q_{0}\right) \prec \omega_{1}=\left(x_{1}, q_{1}\right)
$$

2. The state space $\Omega(\mathscr{W})$ of the hysteron, described by the functions $\Gamma_{r}(x), \Gamma_{l}(x)$, and $\alpha(x, z)$, can be endowed with the following semiorder: relation (10) holds if $z_{0} \leq z_{1}$ where $\alpha\left(x_{0}, z_{i}\right)=q_{i}$ $(i=0,1)$. The correctness of the definition follows from the strict monotonicity of $\alpha$ in its second variable.

Thus, for example, for an arbitrary stop, relation (10) is equivalent to the inequality

$$
x_{0}-q_{0} \leq x_{1}-q_{1} \text {. }
$$

3. Let the function $\mu$ in the definition of the Ishlinskii transducer be nondecreasing, i.e., it induces a nonnegative Lebesgue-Stieltjes measure. Then, for the two states $\omega_{i}=\left(x_{i}, q_{i}(\cdot)\right)$ $(i=0,1)$ of the Ishlinskii transducer, relation (10) is equivalent to the inequality

$$
x_{0}-q_{0}(h) \leq x_{1}-q_{1}(h) \quad \forall h \in \Pi .
$$

4. For the two states $\left.\omega_{i}, q_{i}(\cdot, \cdot)\right)(i=0,1)$ of the Preisach transducer, relation (10) is equivalent to the inequality

$$
q_{0}(\alpha, \beta) \leq q_{1}(\alpha, \beta) \quad \forall(\alpha, \beta) \in \Pi
$$

All the transducers mentioned are directed with respect to corresponding semiorders. For hysterons and Preisachs transducers (in the case of a positive measure $\mu$ ), the fact was pointed out in [4]. As to the Ishlinskii transducer, its directedness immediately follows from that of the stop.

\subsection{Special monotonicity}

Given $x, y \in \mathbb{R}^{n}$, write $x<y$ (or $x \leq y$ ) if the inequality $x_{i}<y_{i}$ (or $x_{i} \leq y_{i}$ ) holds for any index $i(i=1, \ldots, n)$ enumerating the coordinates of the vectors $x, y$. The notation $x \leq j \leq y$ is used for $x \leq y$ and $x_{j}=y_{j}$.

Definition 2: The right-hand side $f(t, x, \xi)$ of equation (7) is called $S_{\prec}$-monotone, if for any positive integer $j \leq n$ and for all $t \in\left[t_{0}, t_{1}\right]$ the relations

$$
x \stackrel{j}{\leq} y, \quad \omega_{1} \prec \omega_{2} \quad\left(\omega_{1}, \omega_{2} \in \Omega(\mathscr{W})\right)
$$


imply the inequality

$$
f_{j}\left(t, x, F\left[\omega_{1}\right]\right) \leq f_{j}\left(t, y, F\left[\omega_{2}\right]\right)
$$

where $f_{j}$ stands for the $j$ th coordinate of the vector $f$ and $F$ is functional (3).

As it can be seen from the definition, the $S_{\prec}$-monotonicity is specified both by the properties of $f$ and functional (3). Despite the cumbersomeness of the definition, it is not difficult to verify the $S_{\prec}$-monotonicity in many situations. Moreover, there are nonlinearities whose $S_{\prec}$ monotonicity can be characterized as a property of the function $f(t, x, \xi)$ only.

Examples: 1. Let $x \in \mathbb{R}$ and $\mathscr{W}$ be the hysteron described by the functions $\Gamma_{l}(x), \Gamma_{r}(x)$, and $\alpha(x, z)$. Then $f(t, x, \xi)$ possesses the property of $S_{\prec}$-monotonicity if $f(t, x, \xi)$ does not decrease in its third variable for all $x$ such that $\alpha\left(x, \Gamma_{l}(x)\right) \geq \alpha\left(x, \Gamma_{r}(x)\right)$, and does not increase in the same variable for all $x$ such that $\alpha\left(x, \Gamma_{r}(x)\right) \geq \alpha\left(x, \Gamma_{l}(x)\right)$.

If $\mathscr{W}$ is a play (ordinary or generalized) or the Preisach transducer then $f(t, x, \xi)$ possesses the $S_{\prec}$-monotonicity whenever $f$ is nondecreasing in $\xi$.

If $\mathcal{W}$ is a stop or an Ishlinskii transducer then $f(t, x, \xi)$ possesses the $S_{\prec}$-monotonicity whenever $f$ is nonincreasing in $\xi$.

2. Let $x$ be a vector in $\mathbb{R}^{n}$ and

$$
f(t, x, \xi)=A(t) x+b(t) \xi
$$

where $A(t)=\left[a_{i j}(t)\right]$ is an $n \times n$-matrix, $b(t)=\left(b_{1}(t), \ldots, b_{n}(t)\right)$ is a vector, and $\xi(t)$ is the value of the output of the Ishlinskii transducer corresponding to the input $x_{1}(t)$. Verify that the $S \prec$ monotonicity of $f$ follows from the following inequalities $\left(t \in\left[t_{0}, t_{1}\right]\right)$ :

where

$$
\left\{\begin{array}{cc}
a_{j}(t) \geq 0 & \text { for } j \neq 1, j \neq i ; \\
b_{i}(t) \leq 0 & \text { for all } i \\
a_{i 1}(t)+E b_{i}(t) \geq 0 & \text { for } i \neq 1
\end{array}\right.
$$

$$
E=\int_{0}^{H} d \mu(h) .
$$

Indeed, for any $i$, the following inequality is valid:

where

$$
\begin{gathered}
\Delta f_{i}:=f_{i}\left(t, x, F\left[\omega_{1}\right]\right)-f_{i}\left(t, y, F\left[\omega_{2}\right]\right) \\
=\sum_{j=1}^{n} a_{j}(t)\left(x_{i}-y_{i}\right)+b_{i}(t) \int_{0}^{H}\left[q_{1}(h)-q_{2}(h)\right] d \mu(h),
\end{gathered}
$$

$$
\omega_{1}=\left(x_{1}, q_{1}(\cdot)\right), \omega_{2}=\left(y_{1}, q_{2}(\cdot)\right) \in \Omega(\mathscr{W}) .
$$

Hence,

$$
\begin{aligned}
\Delta f_{i}= & \sum_{j=2}^{n} a_{i j}(t)(t)\left(x_{i}-y_{i}\right)+\left[a_{i 1}(t)+E b_{i}(t)\right]\left(x_{1}-y_{1}\right) \\
& +b_{i}(t) \int_{0}^{H}\left[q_{1}(h)-x_{1}-q_{2}(h)+y_{1}\right] d \mu(h)
\end{aligned}
$$

and the inequality $\Delta f_{i} \leq 0$ follows from (11)-(13).

Analogously, if $\xi$ is the output of the stop with the threshold values $\pm h$, then inequalities (13), where $E=1$, imply the $S_{\prec}$-monotonicity of the function $A(t) x+b(t) \xi$. 
If $f(t, x, \sigma)$ does not depend on the third variable then its $S_{\prec}$-monotonicity is equivalent to the off-diagonal monotonicity of the function $f(t, x)$ (see [3]). In the monograph [7], the term "condition $W_{+}$" is used.

\section{Differential Inequalities}

\subsection{Basic statements}

In what follows, we will assume that the solutions of problem (7)-(9) are defined on a segment $I=\left[t_{0}, t_{1}\right]$.

Theorem 1: (On differential inequalities) Let $x(t)$ be a solution of problem (7) - (9) and $y(t) \in C^{1}\left(I \rightarrow \mathbb{R}^{n}\right)$. If a transducer $W$ is directed and the right-hand side of equation (7) is $S_{\prec}$ monotone then the relations

$$
\begin{gathered}
y\left(t_{0}\right)=y_{0}<x_{0}, \quad \omega_{1} \prec \omega_{0}, \\
y^{\prime}(t)<f\left(t, y(t), W\left[t_{0}, \omega_{1}\right] y(t)\right) \quad\left(t_{0}<t \leq t_{1}\right),
\end{gathered}
$$

imply the validity of the inequality $y(t)<x(t)$ for all $t \in I$.

Proof: Assume $y(t)<x(t)$ doesn't hold true on $I$. Then there exists $t^{*} \in\left(t_{0}, t_{1}\right)$ and at least one index $i \leq n$ such that

and

$$
y(t)<x(t) \quad t \in\left[t_{0}, t^{*}\right]
$$

$$
y\left(t^{*}\right) \stackrel{i}{\leq} x\left(t^{*}\right)
$$

From the last two inequalities, it follows that

$$
y_{i}^{\prime}\left(t^{*}\right) \geq x_{i}^{\prime}\left(t^{*}\right)
$$

On the other hand, from (14) and (16), by the directedness of $\mathcal{W}$, the relation

$$
\omega_{y}\left(t^{*}\right):=\left(y_{1}\left(t^{*}\right), q_{y}\left(t^{*}\right)\right) \prec\left(x_{1}\left(t^{*}\right), q_{x}\left(t^{*}\right)\right)=: \omega_{x}\left(t^{*}\right)
$$

holds true, where

$$
q_{y}(t)=Z\left[t_{0}, \omega_{1}\right] y_{1}(t) \text { and } q_{x}(t)=Z\left[t_{0}, \omega_{0}\right] x_{1}(t)
$$

(here $x_{1}, y_{1}$ stands for first coordinate of vectors $x$ and $y$ respectively). Hence, by virtue of (17) and taking into account the $S_{\prec}$-monotonicity of $f$ one can obtain that

$$
f_{i}\left(t^{*}, y\left(t^{*}\right), F\left[\omega_{y}\left(t^{*}\right)\right]\right) \leq f_{i}\left(t^{*}, x\left(t^{*}\right), F\left[\omega_{x}\left(t^{*}\right)\right]\right)
$$

$\left(f_{i}\right.$ means $i$ th coordinate of vector function $\left.f\right)$. Further, combining this inequality with $(7)$ and (15), we obtain the inequality $y_{i}^{\prime}\left(t^{*}\right)<x^{\prime}\left(t^{*}\right)$ which contradicts $(18)$. This contradiction proves the Theorem.

It can analogously be proved that, under the assumptions of Theorem 1, the relations

$$
\begin{gathered}
y\left(t_{0}\right)=y_{0}>x_{0}, \quad \omega_{0} \prec \omega_{1}, \\
y^{\prime}(t)>f\left(t, y(t), W\left[t_{0}, \omega_{1}\right] y(t)\right), \quad\left(t_{0}<t \leq t_{1}\right)
\end{gathered}
$$

imply the validity of the inequality $y(t)>x(t)$ for all $t \in I$.

Definition 3: A solution $x^{+}(t)$ of problem (7)-(9) is called maximal if for any solution $x(t)$ of the problem, the inequality $x(t) \leq x^{+}(t)$ holds for all $t \in I$.

A minimal solution $x^{-}(t)$ is defined in the same way as the maximal one.

Theorem 2: If a transducer $W$ is directed and the right-hand side of equation (7) is $S_{\prec}$ - 
monotone then initial value problem (7)-(9) has both maximal and minimal solutions.

Proof: Let $x(t)$ be a solution of problem (7)-(9) and functions $y^{k}(t) \quad(k=1,2, \ldots)$ be solutions of the initial value problems

$$
\left\{\begin{array}{c}
\frac{d}{d t} y^{k}(t)=f\left(t, y^{k}(t), \xi^{k}(t)\right)+\frac{1}{k} \mathbf{e} \\
\xi^{k}(t)=W\left[t_{0}, \omega_{0}\right] y_{1}^{k}(t) ; \quad y^{k}\left(t_{0}\right)=x_{0}
\end{array}\right.
$$

where vector $\mathbf{e} \in \mathbb{R}^{n}$ has all entries equal to 1 . Since the right derivative of the function $x(t)-$ $y^{k}(t)$ at the point $t_{0}$ is strictly negative we obtain that there exists an $s_{k} \in\left(t_{0}, t_{1}\right]$ such that

$$
x(t)<y^{k}(t) \quad\left(t_{0}<t \leq s_{k}\right)
$$

and hence $x_{1}(t)<y_{1}^{k}(t)$. That is why from the directedness of $W$ we get the estimate

$$
\left(x_{1}(t), Z\left[t_{0}, \omega_{0}\right] x_{1}(t)\right) \prec\left(y_{1}^{k}(t), Z\left[t_{0}, \omega_{0}\left[y_{1}^{k}(t)\right) \quad\left(t \in\left[t_{0}, s_{k}\right]\right) .\right.\right.
$$

Since the function $f$ possesses the $S_{\prec}$-monotonicity the same property holds for the right-hand side of (19). Therefore, from relations (20) and (21) and by virtue of Theorem 1 it follows that

$$
x(t)<y^{k}(t) \quad t \in\left[s_{k}, t_{1}\right]
$$

Furthermore, by virtue of $(20)$ we get

$$
x(t)<y^{k}(t) \quad t \in I \quad k=1,2, \ldots
$$

Since the integral operator

$$
J x(t)=x_{0}+\int_{t_{0}}^{t} f\left(s, x(s), W\left[t_{0}, \omega_{0}\right] x_{1}(s)\right) d s
$$

corresponding to problem (7)-(9) is completely continuous on the space $C$, we conclude that the sequence of solutions

$$
y^{k}(t)=J y^{k}(t)+\frac{t-t_{0}}{k} \mathbf{e}
$$

of problem (19) may be regarded as uniformly convergent on the segment $I$ to a continuous function $y^{*}(t)$. Passing to the limit in (22) and (23) (for $k \rightarrow \infty$ ), we obtain the estimate

$$
x(t) \leq y^{*}(t)=J y^{*}(t), \quad t \in I \text {. }
$$

Since $x(t)$ was an arbitrary solution of problem (7)-(9) it follows that $y^{*}(t)=x^{+}(t)$ is a maximal solution. Existence of a minimal solution can be established in the same way.

The following theorem is proved similarly to Theorem 2 .

Theorem 3: Let the assumptions of Theorem 1 hold. Then the relations

$$
\begin{gathered}
y\left(t_{0}\right)=y_{0} \leq x_{0}, \quad \omega_{1} \prec \omega_{0} ; \\
y^{\prime}(t) \leq f\left(t, y(t), W\left[t_{0}, \omega_{1}\right] y(t)\right), \quad\left(t_{0}<t<1_{1}\right)
\end{gathered}
$$

imply the validity of the inequality $y(t) \leq x^{+}(t)$ for all $t \in I$.

\subsection{An example}

As was indicated above, if $x$ is a scalar and $\mathcal{L}$ is a play, then the $S_{\prec}$-monotonicity of the function $f(t, x, \xi)$ is equivalent to that the function is nondecreasing in its third variable in the region $(x, \xi) \in \Omega(\ell)$. A violation of the $S \prec$-monotonicity may lead to the absence of maximal or minimal solutions among solutions of the initial value problem.

Consider the following example. Let $\mathcal{L}$ be the play described by the functions $\Gamma_{r}(x)=x$ and 
$\Gamma_{l}(x)=x+2$. Take a bounded continuous function $f(x, \xi)$ such that

$$
\begin{gathered}
f(x, x)=\sqrt{\max \{0,1-|x-1|\}} ; \quad f(x, x+2)=-\sqrt{2} \\
f(x, z)=-\sqrt{|2-x|} ; \quad f(x, 0)=x / \sqrt{2} .
\end{gathered}
$$

Therefore, the initial value problem

$$
x^{\prime}(t)=f\left(x(t), W_{\ell}\left[0, \omega_{0}\right] x(t)\right) ; \quad \omega_{0}=(0,0), \quad x(0)=0
$$

has solutions, and they are well-defined for all $t \geq 0$. The set of the solutions is described as follows: there is the identically zero solution, and other ones are given by

$$
x(t ; a, b)=\left\{\begin{array}{cc}
0 & \text { for } 0 \leq t \leq a \\
(t-a)^{2} / 4 & \text { for } a \leq t \leq a+2 \\
2-(t-a-4)^{2} / 4 & \text { for } a+2 \leq t \leq a+4 \\
2 & \text { for } a+4 \leq t \leq a+b+4 \\
2-(t-a-b-4)^{2} / 4 & \text { for } a+b+4 \leq t \leq a+b+4+2 \sqrt{2} \\
-\sqrt{2}(t-a-b-4-2 \sqrt{2}) & \text { for } t \geq a+b+4+2 \sqrt{2},
\end{array}\right.
$$

where $a, b$ are arbitrary nonnegative numbers ( $b$ may take an $\infty$-value). There are no other solutions of problem (25). Obviously,

$$
x^{-}(t)=\min _{a, b} x(t ; a, b)=\left\{\begin{array}{cc}
0 & \text { for } 0 \leq t \leq 4+2 \sqrt{2} \\
-\sqrt{2}(t-4-2 \sqrt{2}) & \text { for } t \geq 4+2 \sqrt{2}
\end{array}\right.
$$

and the function $x^{-}(t)$ is not a solution of problem (25).

\subsection{Possible extensions}

The assumption on smoothness of the function $y(t)$ used in the previous theorems is not essential. One may instead assume the continuity of $y(t)$. In this case, one should only substitute any left-hand Dini's derivative (see [7]) of $y(t)$ for the derivative of the function in inequality (15); the derivative in inequality (24) may be replaced with an arbitrary chosen Dini's derivatives. Besides, the functions $x(t)$ and $y(t)$ participating in Theorems 1-3 may be taken absolutely continuous and equation (7) along with inequalities (15) and (24) may be assumed to hold true almost everywhere on the segment $I$.

\subsection{On delayed systems}

The scheme proposed above is applicable to other classes of functional differential equations. Particularly, it can be applied to equations with finite delay:

$$
x^{\prime}(t)=f(t, x(t), x(t-\tau)) \text {. }
$$

Theorem 4: Let $\tau>0$, function $x(t)$ satisfy equation (26) and function $y(t)$ obey the inequali ty $y^{\prime}(t)<f(t, y(t), y(t-\tau))$ for all $t \in\left[t_{0}+\tau, t_{1}\right]$. In addition, suppose that the relation

$$
y(t)<x(t)
$$

holds true for any $t \in\left[t_{0}, t_{0}+\tau\right]$. If $f(t, x, \xi): \mathbb{R}^{3} \rightarrow \mathbb{R}$ does not decrease with respect to $\xi$ then inequality (27) is also valid on $\left[t_{0}, t_{1}\right]$. 
Proof: Consider a "delayed" dynamical system $D_{\tau}$ with the state space

$$
\Omega\left(D_{\tau}\right)=\{(x, q(\cdot)) \mid q(\cdot) \in C[0, \tau], q(\tau)=x\} .
$$

The transition operator $Z_{\tau}$ of transducer $D_{\tau}$ assigns the pair $\left\{\omega_{0}, x(t)\right\}$ (of course, $x\left(t_{0}\right)=x_{0}$ ) to the function $q(\cdot)(t)$ given by

$$
q(\eta)(t)=Z_{\tau}\left[t_{0},\left(x_{0}, q_{0}(\eta)\right)\right] x(t)=\left\{\begin{array}{cc}
q_{0}\left(\eta+t-t_{0}\right) & \text { for } \eta+t-t_{0}<\tau \\
x(\eta+t-\tau) & \text { for } \eta+t-t_{0} \geq \tau .
\end{array}\right.
$$

The output of $D_{\tau}$ is defined as

$$
F[\omega(t)]=F[x, q(\cdot)]=q(0) .
$$

With these definitions in mind, initial value problem (7)-(9) is equivalent to (26) combined with initial value

$$
x\left(t_{0}+\eta\right) \equiv q_{0}(\eta), \quad \eta \in[0, \tau] .
$$

Introduce semiorder in $\Omega\left(D_{\tau}\right)$ as

$$
\omega_{0}\left(x_{0}, q_{0}(\cdot)\right) \prec \omega_{1}\left(x_{1}, q_{1}(\cdot)\right),
$$

whenever $q_{0}(\eta) \leq q_{1}(\eta)$ for all $\eta \in[0, \tau]$.

After that it is not difficult to verify the validity of all assumptions of Theorem 1; Theorem 4 becomes the direct consequence.

\section{References}

[1] Chernorutskii, V.V., Krasnosel'skii, M.A., and Vladimirov, A.A., The Cauchy problem for systems with hysteresis, Russian Aca. Sci. Dokl. Math 48:3 (1994), 502-506.

[2] Kalman, R.E., Falb, P.L., and Arbib, M., Topics in Mathematical System Theorem, McGraw-Hill, New York 1969.

[3] Krasnosel'skii, M.A., The Operator of Translation along the Trajectories of Differential Equations, Trans. of Mathematical Monographs 19, American Math Society, Providence, RI 1968.

[4] Krasnosel'skii, M.A. and Pokrovskii, A.V., Systems with Hysteresis, Springer-Verlag, Berlin 1989.

[5] Lakshmikantham, V. and Leela, S., Differential and Integral Inequalities. Theory and Applications, Academic Press, New York 1969.

[6] Mayergoyz, I.D., Mathematical Models of Hysteresis, Springer-Verlag, New York 1991.

[7] Szarski, J., Differential Inequalities, PWN, Warsaw 1965.

[8] Zadeh, L.A. and Desoer, C.A., Linear System Theory: the State Space Approach, McGraw-Hill, New York 1963. 


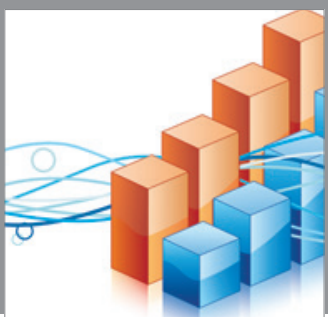

Advances in

Operations Research

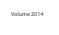

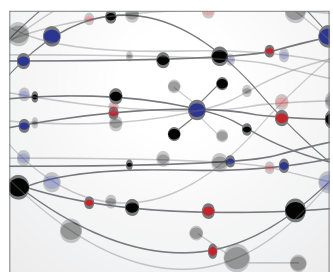

\section{The Scientific} World Journal
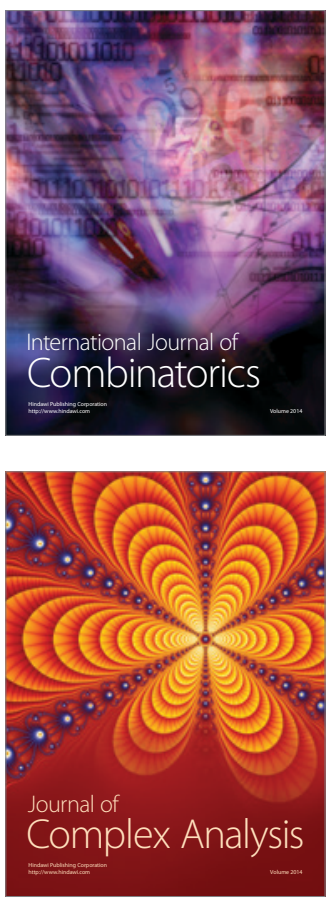

International Journal of

Mathematics and

Mathematical

Sciences
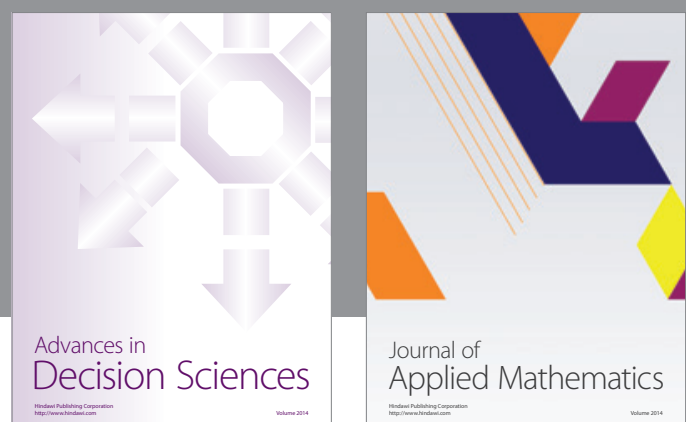

Journal of

Applied Mathematics
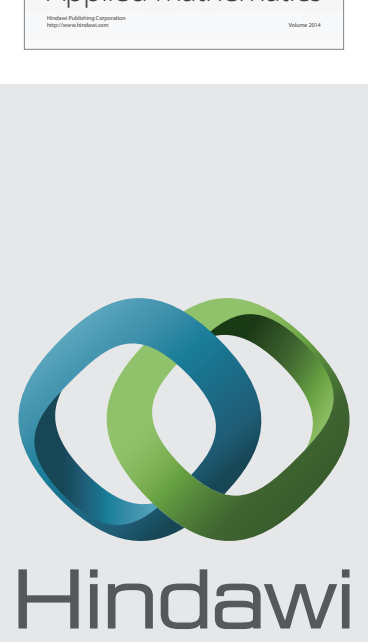

Submit your manuscripts at http://www.hindawi.com
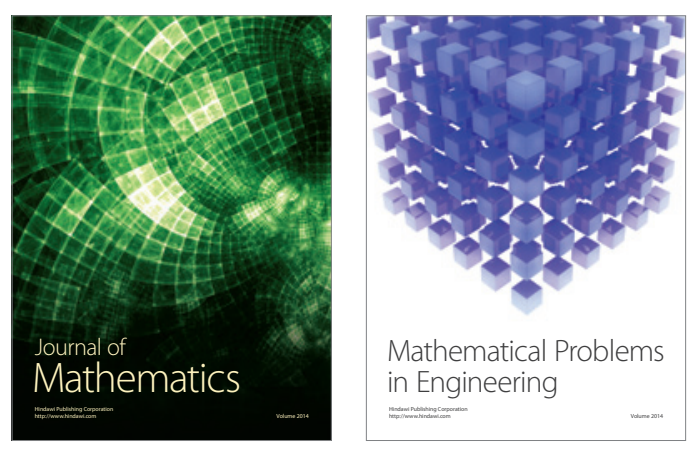

Mathematical Problems in Engineering
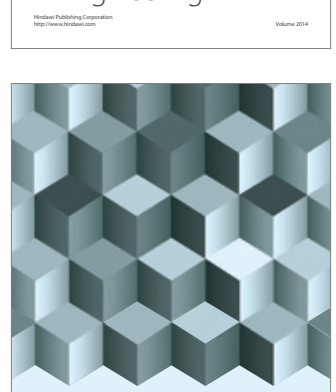

Journal of

Function Spaces
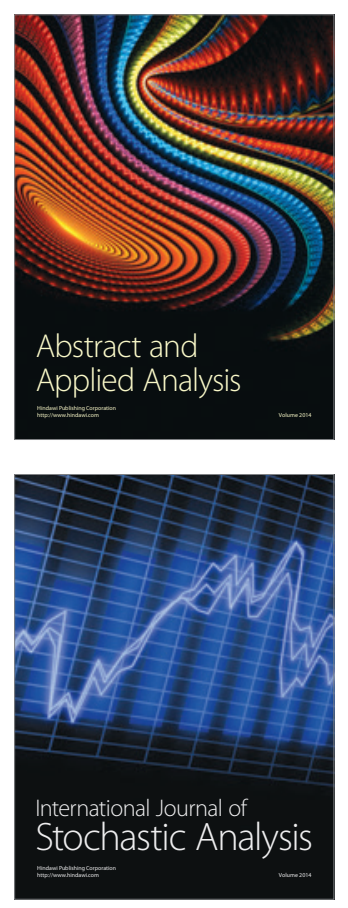

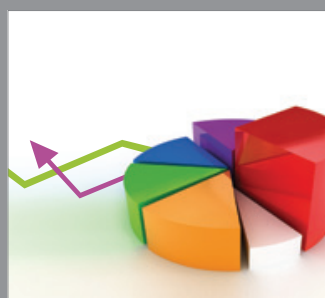

ournal of

Probability and Statistics

Promensencen
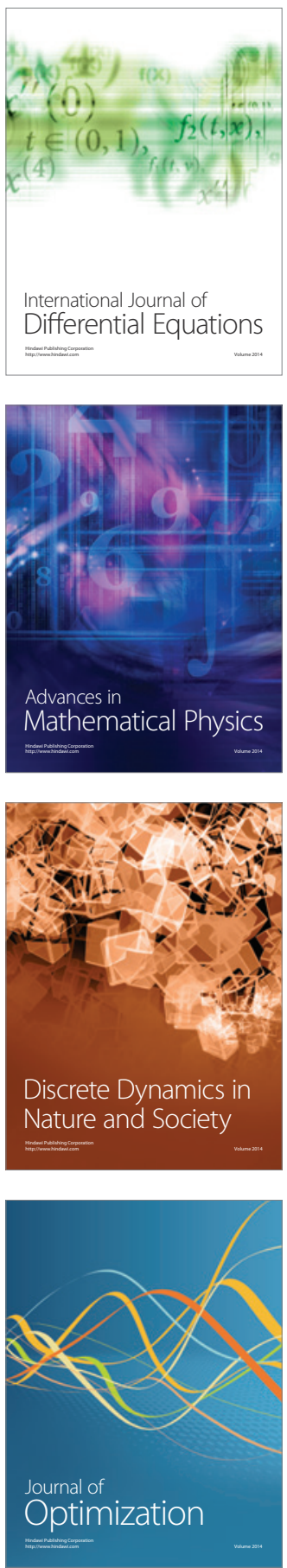